\title{
The contribution of effective quantum gravity to the high energy scattering in the framework of modified perturbation theory and one loop approximation
}

\author{
Nguyen Suan Han ${ }^{1,2, a}$, Do Thu Ha ${ }^{2,4, b}$, Nguyen Nhu Xuan ${ }^{3, c}$ \\ ${ }^{1}$ Bogoliubov Laboratory of Theoretical Physics, JINR, Dubna 141980, Russia \\ ${ }^{2}$ Hanoi University of Science, Ha Noi, Vietnam \\ ${ }^{3}$ Department of Physics, Le Qui Don University, Hanoi, Vietnam \\ ${ }^{4}$ Hanoi University of Natural Resources and Environment, Ha Noi, Vietnam
}

Received: 4 April 2019 / Accepted: 29 September 2019 / Published online: 10 October 2019

(C) The Author(s) 2019

\begin{abstract}
The asymptotic behavior of the scattering amplitude for two scalar particles at high energies with fixed momentum transfers is studied. The study is done within the effective theory of quantum gravity based on quasi-potential equation. By using the modified perturbation theory, a systematic method is developed to find the leading eikonal scattering amplitudes together with corrections to them in the one-loop gravitational approximation. The relation is established and discussed between the solutions obtained by means of the operator and functional approaches applied to quasi-potential equation. The first non-leading corrections to the leading eikonal amplitude are found.
\end{abstract}

\section{Introduction}

The asymptotical behavior of the scattering amplitude at high energy for all types of interactions including gravitational interaction is one of the central problems of elementary particle physics. From the standard method of quantum field theory it follows that calculations based on perturbation theory are suitable when the energy of individual particles is not very high and the effective coupling constant is not very large. When the energy is increased the effective coupling constant also increases so that the corrections calculated by perturbation theory play a crucial role. The gravitational scattering occurs at energies $\sqrt{s}=2 E<<M_{P L}-$ and is described by "effective field theory", where $s$ is the squared energy of the center of mass, $M_{P L}$ is the Planck mass, $G$ is the universal gravitational constant, which is characterized by the

\footnotetext{
a e-mails: suanhan@hus.edu.vn; suan@theor.jinr.ru

be-mail: thuhahunre@gmail.com

c e-mail: xuannn@mta.edu.vn
}

effective coupling constant $\alpha_{G}=G s / \hbar \geq 1$ and makes any simple perturbation expansion unwarranted. Comparison of the results of different approaches tackling this problem has shown to coincide in the leading order of approximation that has a semi-classical effective metric interpretation, while the most of them fail in providing the non-leading terms [117]. The determination of these corrections to gravitational scattering is currently an open problem. These corrections play a crucial role in such problems as strong gravitational forces near the black hole, string modification of the gravitational theory and some other effects of quantum gravity $[3-9,12,13]$.

In the framework of standard field theory and the highenergy scattering, the different methods have been developed to investigate the asymptotic behavior of individual Feynman diagrams and their subsequent summation. The calculations of the eikonal diagrams in the case of gravity run similarly to the analogous calculations in QED. The calculations of eikonal capture the leading behavior of each order in perturbation theory, but the sum of leading terms is subdominant to the neglected term by this approximation. The reliability of eikonal amplitude for gravity is uncertain. An approach that has probed the first of these features with some success is the one that based on the reggeized string exchange amplitudes with subsequent reduction to the gravitational eikonal limit including the leading order corrections [18]. In articles [19-21] the high energy scattering amplitude of two "nucleons" in the quantum gravity is constructed by extending the functional integration method [22-28] which has been used effectively in quantum electrodynamics [29-38]. A straightline path approximation was used to calculate the functional integrals which occur. It is shown that in the limit of asymptotically high energy $s \rightarrow \infty$ and at fixed $t$-momentum transfer, the elastic scattering amplitude of two "nucleons" has 
the form of a Glauber representation with an eikonal function depending on the energy. A similar result is obtained by the "shock-wave method" proposed by 't Hooft $[1,2]$, by the method of effective topological theory in the Planck limit proposed by Verlinde and Verlinde [14] and by summation of Feynman diagrams in the eikonal approximation [15]. The main advantage of the proposed approach over the other approaches known is the possibility to perform calculations in a compact form. In doing so, the correct structure of the Green's function and amplitudes etc. is not destroyed by approximations in the process of the calculations.

It should be emphasized that in the framework of functional integrals the modified perturbation theory proposed by Fradkin in [23-25] leads to results that go well beyond those of ordinary perturbation theory. This method is especially fruitful in quantum electrodynamics since even in the first approximation of the modified perturbation theory the contribution of both the virtual and the real soft photons can be completely summed. In particular, it was shown that the first approximation of the modified perturbation theory is already sufficient to obtain the correct asymptotic behavior for the Green's functions and the cross sections of processes in quantum electrodynamics [23-26] in the so-called log$\log$ approximation. These general results allow to solve the problem of finding the quantum Green's functions and cross sections of processes with allowance for the radiative corrections in electrodynamics in the presence of a real external field without resorting to perturbation theory.

The purpose of the present paper is to develop a systematic scheme based on modified perturbation theory to find the correction terms to the leading eikonal amplitude by solving the Logunov-Tavkhelidze quasi-potential equation [39-50]. In spite of the lack of a clear relativistic covariance, the quasipotential method keeps all information about properties of scattering amplitude which could be received from the general principle of quantum field theory [39]. Therefore, at high energies one can investigate analytical properties of the scattering, its asymptotic behavior and some regularities of a potential scattering etc., exactly, as it has been done in the usual S-matrix theory. The choice of this approach is dictated also by the following reasons: (i) in the framework of the quasi-potential approach, the eikonal amplitude has a rigorous justification in quantum field theory [43]; (ii) in the case of smooth potentials, it was shown that a relativistic quasipotential and the Schrodinger equations lead to qualitatively identical results $[42,46]$.

This paper is organized as follows: In the second section we briefly introduce the Logunov-Tavkhelidze quasipotential equation, and rewrite this equation on a mass shell in the operator form, after defining an appropriate pseudodifferential operator. The solution of this equation is presented in an exponent form which is favorable to modify the perturbation method in the framework of functional inte- grals in the third section. The asymptotic behavior of scattering amplitude at high energies and fixed momentum transfer is also considered and a systematic scheme of finding the leading eikonal scattering amplitudes and its corrections are constructed in the fourth section. The lowest-order approximation of the modified perturbation theory is the leading eikonal scattering amplitude in the linearized quantum gravity. The first correction to leading eikonal amplitude is also found. The quasi-potential used here corresponds to virtual particles between two "nucleons". The fifth section is devoted to calculating the one-loop leading eikonal scattering amplitude and its first correction to leading amplitude in the effective theory of quantum gravity. The new results here for the non-analytic and non-local contributions were discussed and included in the calculation of the behavior scattering amplitude by using the Newtonian potential with low-energy. The leading one loop gravitational corrections was obtained from S-matrix in quantum gravity. Finally, we discuss the obtained results and possible generalization of this approach.

\section{Two-particle quasi-potential equation in an operator form}

For simplicity, we shall first consider the elastic scattering of two scalar "nucleons" with the interaction Lagrangian $L_{i n t}=$ $g \varphi^{2}(x) \phi(x)$. The results will be generalized to the case of a scalar nucleon interacting with a neutral vector and a graviton fields later. For two scalar particle scattering amplitude the quasi-potential equation with local quasi-potential has the form [47]:

$$
\begin{aligned}
T\left(\mathbf{p}, \mathbf{p}^{\prime} ; s\right)= & g V\left(\mathbf{p}-\mathbf{p}^{\prime} ; s\right) \\
& +g \int d \mathbf{q} V(\mathbf{p}-\mathbf{q} ; s) K\left(\mathbf{q}^{2}, s\right) T\left(\mathbf{q}, \mathbf{p}^{\prime} ; s\right),
\end{aligned}
$$

where $K\left(\mathbf{q}^{2}, s\right)=\frac{1}{\sqrt{q^{2}+m^{2}}} \frac{1}{q^{2}+m^{2}-\frac{s}{4}-i \varepsilon}$ is the kernel, $s=$ $4 E^{2}=4\left(\mathbf{p}^{2}+m^{2}\right)=4\left(\mathbf{p}^{\prime}+m^{2}\right)$ is the energy and $\mathbf{p}, \mathbf{p}^{\prime}$ are the relativistic momentums of two particles in the center of the mass reference frame in the initial and final states respectively. Equation (1) is one of the possible generalizations of the Lippman-Schwinger equation for the case of relativistic quantum field theory. The quasi-potential in Eq. (1) is a complex function of the energy and relativistic momenta. The quasi-potential equation simplifies considerably if $V(r, s)$ is a function that depends only of the relative momenta and the total energy i.e. if the quasi-potential is local. ${ }^{1}$ The existence

\footnotetext{
${ }^{1}$ Since the total energy as an external parameter of this equation, the "local" here has direct meaning and it can appear in a three-dimensional $\delta$ function in the quasi-potential in the coordinate representation.
} 
of the local quasi-potential has been well proven rigorously in the weak coupling case [45] and a method has been specified for constructing it. The local potential constructed in this manner gives a solution of Eq. (1) that is equal to the physical amplitude on the mass shell $[39,40,42,45]$.

Performing the Fourier transformations

$$
\begin{aligned}
V\left(\mathbf{p}-\mathbf{p}^{\prime} ; s\right) & =\frac{1}{(2 \pi)^{3}} \int d \mathbf{r} e^{i\left(\mathbf{p}-\mathbf{p}^{\prime}\right) \mathbf{r}} V(\mathbf{r} ; s), \\
T\left(\mathbf{p}, \mathbf{p}^{\prime} ; s\right) & =\int d \mathbf{r} d \mathbf{r}^{\prime} e^{i\left(\mathbf{p r}-\mathbf{p}^{\prime} \mathbf{r}^{\prime}\right)} T\left(\mathbf{r}, \mathbf{r}^{\prime} ; s\right),
\end{aligned}
$$

and substituting Eqs. (2) into (1), we obtain

$$
\begin{aligned}
T\left(\mathbf{r}, \mathbf{r}^{\prime} ; s\right)= & \frac{g}{(2 \pi)^{3}} V(\mathbf{r} ; s) \delta^{(3)}\left(\mathbf{r}-\mathbf{r}^{\prime}\right) \\
& +\frac{g}{(2 \pi)^{3}} \int d \mathbf{q} K\left(q^{2} ; s\right) V(\mathbf{r} ; s) e^{-i \mathbf{q r}} \\
& \times \int d \mathbf{r}^{\prime \prime} e^{i \mathbf{q} \mathbf{r}^{\prime \prime}} T\left(\mathbf{r}^{\prime \prime}, \mathbf{r}^{\prime} ; s\right)
\end{aligned}
$$

Introducing the representation

$T\left(\mathbf{r}, \mathbf{r}^{\prime} ; s\right)=\frac{g}{(2 \pi)^{3}} V(\mathbf{r} ; s) F\left(\mathbf{r}, \mathbf{r}^{\prime} ; s\right)$,

we find

$$
\begin{aligned}
F\left(\mathbf{r}, \mathbf{r}^{\prime} ; s\right)= & \delta^{(3)}\left(\mathbf{r}-\mathbf{r}^{\prime}\right)+\frac{g}{(2 \pi)^{3}} \int d \mathbf{q} K\left(\mathbf{q}^{2} ; s\right) e^{-i \mathbf{q r}} \\
& \times \int d \mathbf{r}^{\prime \prime} e^{i \mathbf{q} \mathbf{r}^{\prime \prime}} V\left(\mathbf{r}^{\prime \prime} ; s\right) F\left(\mathbf{r}^{\prime \prime}, \mathbf{r}^{\prime} ; s\right) .
\end{aligned}
$$

Defining the pseudo-differential operator $\widehat{L_{r}}=K\left(-\nabla_{\mathbf{r}}{ }^{2} ; s\right)$, then

$$
\begin{aligned}
K(\mathbf{r} ; s) & =\int d \mathbf{q} K\left(\mathbf{q}^{2} ; s\right) e^{-i \mathbf{q r}} \\
& =K\left(-\nabla_{r} ; s\right) \int d \mathbf{q} e^{-i \mathbf{q} \mathbf{r}}=\widehat{L_{r}}(2 \pi)^{3} \delta^{(3)}(\mathbf{r})
\end{aligned}
$$

After some simple transformations, Eq. (3) is rewritten in an operator form as:

$$
F\left(\mathbf{r}, \mathbf{r}^{\prime} ; s\right)=\delta^{(3)}\left(\mathbf{r}-\mathbf{r}^{\prime}\right)+g \widehat{L_{r}}\left[V(\mathbf{r}, s) F\left(\mathbf{r}, \mathbf{r}^{\prime}, s\right)\right] .
$$

Equation (7) is the operator form of the LogunovTavkhelizde equation [47].

Within the framework of the quasi-potential approach, the potential is defined by expanding it into infinite series in order of the interaction constant $g$. It corresponds to the expansion of perturbation amplitudes on the mass shell. The approximate solution of Eq. (5) is found in the lowest order of the quasi-potential. Using this method, the relativistic eikonal expression of the scattering amplitude was found in quantum field theory with large energy and small momentum transfer [43].

\section{Modified perturbation theory}

In quantum field theory, scattering problems are mostly solved by perturbation expansion. However, in some cases, in order to solve the problem smoothly, one can improve this method in an expansion that is called the modified perturbation method proposed by Fradkin ${ }^{2}$ in the framework of functional integrals [23-25], which goes well beyond the ordinary perturbation theory. Apply it to Eq. (7), we can write the solution of this equation in the symbolic form

$$
F\left(\mathbf{r}, \mathbf{r}^{\prime} ; s\right)=\frac{1}{(2 \pi)^{3}} \int d \mathbf{k} \exp [W(\mathbf{r} ; \mathbf{k} ; s)] e^{-i \mathbf{k}\left(\mathbf{r}-\mathbf{r}^{\prime}\right)}
$$

Substituting Eqs. (8) into (7), we obtain an equation for the function $W(\mathbf{r}, \mathbf{k} ; s)$

$$
\begin{aligned}
& \exp W(\mathbf{r} ; \mathbf{k} ; s) \\
& \quad=1+g \widehat{L_{r}}\{V(\mathbf{r}, s) \exp [W(\mathbf{r}, \mathbf{k} ; s)-i \mathbf{k r}]\} e^{i \mathbf{k r}} .
\end{aligned}
$$

Using the idea of the modified perturbation theory in exponent function, we can write the function $W(\mathbf{r}, \mathbf{k} ; s)$ as an expansion in series in the coupling constant $g$

$W(\mathbf{r} ; \mathbf{k} ; s)=\sum_{n=1}^{\infty} g^{n} W_{n}(\mathbf{r} ; \mathbf{k} ; s)$.

Substituting Eqs. (10) into (9) and using Taylor expansion, the 1.h.s of Eq. (9) is rewritten as

$$
1+\sum_{n=1}^{\infty} g^{n} W_{n}+\frac{1}{2 !}\left(\sum_{n=1}^{\infty} g^{n} W_{n}\right)^{2}+\frac{1}{3 !}\left(\sum_{n=1}^{\infty} g^{n} W_{n}\right)^{3}+\cdots
$$

\footnotetext{
2 The interpretation of the perturbation theory from the view-point of the diagrammatic technique is as follows. The typical Feynman denominator of the standard perturbation theory is of the form $(A)$ : $\left(p+\sum q\right)^{2}+m^{2}-i \varepsilon=p^{2}+m^{2}+2 p \sum q+\left(\sum q\right)^{2}$, where $p$ is the external momentum of the scalar (spinor) particle, and the $q^{\prime} s$ are virtual momenta of radiation quanta. The lowest order approximation $(A)$ of modified theory is equivalent to summing all Feynman diagrams with the replacement: $\left(\sum q\right)^{2}=\sum(q)^{2}$ in each denominator $(A)$. The modified perturbation theory thus corresponds to a small correlation of the radiation quanta: $\mathbf{q}_{\mathbf{i}} \mathbf{q}_{\mathbf{j}}=\mathbf{0}$ and is often called the $\mathbf{q}_{\mathbf{i}} \mathbf{q}_{\mathbf{j}}$-approximation. In the framework of functional integration this approximation is called the straight-line path approximation i.e high-energy particles move along Feynman paths, which are practically rectilinear.
} 
and the r.h.s of Eq. (9) has the form

$$
\begin{aligned}
1 & +g\left\{\hat{L}_{r}[V(\mathbf{r} ; s)\right. \\
& \left.\times\left(1+\sum_{n=1}^{\infty} g^{n} W_{n}+\frac{1}{2 !}\left(\sum_{n=1}^{\infty} g^{n} W_{n}\right)^{2}+\cdots\right)\right] \\
& +V(\mathbf{r} ; s)\left[1+\sum_{n=1}^{\infty} g^{n} W_{n}+\frac{1}{2 !}\left(\sum_{n=1}^{\infty} g^{n} W_{n}\right)^{2}+\cdots\right] \\
& \times K(\mathbf{k} ; s)\} .
\end{aligned}
$$

Using Eqs. (11) and (12) and comparing with the both sides of Eq. (9) in order of the coupling constant $g$, we derive the first, second, and third order approximation terms, respectively

$$
\begin{aligned}
& W_{1}(\mathbf{r} ; \mathbf{k} ; s)=\int d \mathbf{q} V(\mathbf{q} ; s) K\left[(\mathbf{k}+\mathbf{q})^{2} ; s\right] e^{-i \mathbf{q r}}, \\
& W_{2}(\mathbf{r} ; \mathbf{k} ; s)=-\frac{W_{1}^{2}(\mathbf{r} ; \mathbf{k} ; s)}{2 !} \\
& \quad+\frac{1}{2} \int d \mathbf{q}_{1} d \mathbf{q}_{2} V\left(\mathbf{q}_{1} ; s\right) V\left(\mathbf{q}_{2} ; s\right) K\left[\left(\mathbf{k}+\mathbf{q}_{1}+\mathbf{q}_{2}\right)^{2} ; s\right] \\
& \quad \times\left[K\left(\mathbf{k}+\mathbf{q}_{1} ; s\right)+K\left[\mathbf{k}+\mathbf{q}_{2} ; s\right]\right] e^{-i \mathbf{q}_{1} \mathbf{r}-i \mathbf{q}_{2} \mathbf{r}}, \\
& W_{3}(\mathbf{r} ; \mathbf{k} ; s)=-\frac{W_{1}^{2}(\mathbf{r} ; \mathbf{k} ; s)}{3 !} \\
& \quad+\int d \mathbf{q}_{1} d \mathbf{q}_{2} d \mathbf{q}_{3} V\left(\mathbf{q}_{1} ; s\right) V\left(\mathbf{q}_{2} ; s\right) V\left(\mathbf{q}_{3} ; s\right) K\left[\left(\mathbf{k}+\mathbf{q}_{1}\right)^{2} ; s\right] \\
& \quad \times K\left[\left(\mathbf{k}+\mathbf{q}_{1}+\mathbf{q}_{2}\right)^{2} ; s\right] K\left[\left(\mathbf{k}+\mathbf{q}_{1}+\mathbf{q}_{2}+\mathbf{q}_{3}\right)^{2} ; s\right] \\
& \quad \times \exp \left(-i \mathbf{q}_{1} \mathbf{r}-i \mathbf{q}_{2} \mathbf{r}-i \mathbf{q}_{3} \mathbf{r}\right) .
\end{aligned}
$$

etc.

Restricting the treatment to only $W_{1}(\mathbf{r}, \mathbf{k} ; s)$ instead of $W(\mathbf{r}, \mathbf{k} ; s)$ in Eq. (8) we obtain from Eqs. (2), (4) and (8) approximate expression for the scattering amplitude

$$
T_{1}\left(\mathbf{p}, \mathbf{p}^{\prime} ; s\right)=\frac{g}{(2 \pi)^{3}} \int d \mathbf{r} e^{i\left(\mathbf{p}-\mathbf{p}^{\prime}\right) \mathbf{r}} V(\mathbf{r}, s) e^{g W_{1}(\mathbf{r}, \mathbf{p}, s)} .
$$

To establish the meaning of this approximation, we expand $T_{1}(\mathbf{r}, \mathbf{k} ; s)$ in series in the coupling constant $g$ :

$$
\begin{aligned}
& T_{1}^{(n+1)}\left(\mathbf{p}, \mathbf{p}^{\prime} ; s\right)=\frac{g^{n+1}}{n !} \int d \mathbf{q}_{1} \cdots d \mathbf{q}_{n} V\left(\mathbf{q}_{1} ; s\right) \cdots V\left(\mathbf{q}_{n} ; s\right) \\
& \quad \times V\left(\mathbf{p}-\mathbf{p}^{\prime}-\sum_{i=1}^{n} q_{i} ; s\right) \prod_{i=0}^{n} K\left[\left(\mathbf{q}_{i}+\mathbf{p}^{\prime}\right)^{2} ; s\right] .
\end{aligned}
$$

and compare it with the $(n+1)-t h$ iteration term of exact Eq. (1)

$T^{(n+1)}\left(\mathbf{p}, \mathbf{p}^{\prime} ; s\right)=\int d \mathbf{q}_{1} \cdots d \mathbf{q}_{n} V\left(\mathbf{q}_{1} ; s\right) \cdots V\left(\mathbf{q}_{n} ; s\right)$

$$
\begin{aligned}
& \times V\left(\mathbf{p}-\mathbf{p}^{\prime}-\sum_{i=1}^{n} q_{i} ; s\right) \\
& \times \sum_{p} K\left[\left(\mathbf{q}_{1}+\mathbf{p}^{\prime}\right)^{2} ; s\right] K\left[\left(\mathbf{q}_{1}+\mathbf{q}_{2}+\mathbf{p}^{\prime}\right)^{2} ; s\right] \cdots \\
& \times K\left[\left(\sum_{i=1} \mathbf{q}_{i}+\mathbf{p}^{\prime}\right)^{2} ; s\right]
\end{aligned}
$$

where $\sum_{p}$ is the sum over the permutations of the momenta $\mathbf{p}_{1}, \mathbf{p}_{2} \cdots \mathbf{p}_{n}$.

It is readily seen from Eqs. (17) and (18) that our approximation in the case of the Lippmann-Schwinger equation is identical with the $\mathbf{q}_{i} \mathbf{q}_{j}=0$ approximation, in accordance with which terms of the type $\mathbf{q}_{i} \mathbf{q}_{j}=0, i \neq j$ are ignored in the "nucleon propagators".

\section{Asymptotic behavior of the scattering amplitude at high energy}

In this section the solution of the Logunov-Tavkhelidze quasi-potential equation obtained in the previous section for the scattering amplitude can be used to find the asymptotic behavior amplitude in the high energy $s \rightarrow \infty$ and fixed $t$-momentum transfer. In asymptotic expansions, we shall retain both the principal term and the next order term, using the formula

$e^{W\left(\mathbf{r}, \mathbf{p}^{\prime} ; s\right)}=e^{W_{1}\left(\mathbf{r}, \mathbf{p}^{\prime} ; s\right)}\left[1+g^{2} W_{2}\left(\mathbf{r}, \mathbf{p}^{\prime} ; s\right)+\cdots\right]$,

where $W_{1}(\mathbf{r}, \mathbf{k} ; s)$ and $W_{2}(\mathbf{r}, \mathbf{k} ; s)$ were determined by Eqs. (13) and (14), respectively.

Take the $z$ axis along the $\left(\mathbf{p}+\mathbf{p}^{\prime}\right)$ momentum of the incident particles and use Mandelstam variables, we have

$\boldsymbol{\Delta}_{\perp} \cdot \mathbf{n}_{z}=0, \quad t=-\Delta_{\perp}^{2}, \quad \mathbf{p}-\mathbf{p}^{\prime}=\Delta_{\perp}$.

Noting

$$
\begin{aligned}
K & \left(\mathbf{p}+\mathbf{p}^{\prime} ; s\right) \\
& =\left.\frac{1}{\sqrt{\left(\mathbf{p}+\mathbf{p}^{\prime}\right)^{2}+m^{2}}} \frac{1}{\left(\mathbf{p}+\mathbf{p}^{\prime}\right)^{2}-\frac{s}{4}+m^{2}-i \varepsilon}\right|_{s \rightarrow \infty, t-\text { fixed }} \\
& =\frac{2}{s\left(q_{z}^{2}-i \varepsilon\right)}\left[1-\frac{3 q_{z}^{2}+\mathbf{q}_{\perp}^{2}+\mathbf{q}_{\perp} \Delta_{\perp}}{\sqrt{s}\left(q_{z}-i \epsilon\right)}\right]+O\left(\frac{1}{s^{2}}\right),(21)
\end{aligned}
$$

Substitute Eqs. (16) into (12) and (13), we get

$$
\begin{aligned}
& W_{1}=\left(\frac{W_{10}}{s}\right)+\left(\frac{W_{11}}{s \sqrt{s}}\right)+O\left(\frac{1}{s^{2}}\right), \\
& W_{2}=\left(\frac{W_{20}}{s^{2} \sqrt{s}}\right)+O\left(\frac{1}{s^{3}}\right),
\end{aligned}
$$


where the $W_{10}, W_{11}$ and $W_{20}$ terms are calculated in detail in Ref. [21]

$$
\begin{aligned}
W_{10}= & 2 \int d \mathbf{q} V(\mathbf{q} ; s) \frac{e^{i \mathbf{q r}}}{\left(q_{z}^{2}-i \varepsilon\right)^{2}} \\
= & 2 i \int_{-\infty}^{z} d z^{\prime} V\left(\sqrt{\mathbf{q}_{\perp}^{2}+z^{\prime 2}} ; s\right), \\
W_{11}= & -2 \int d \mathbf{q} V(\mathbf{q} ; s) e^{-i \mathbf{q r}} \frac{3 q_{z}^{2}+\mathbf{q}_{\perp}^{2}+\mathbf{q}_{\perp} \triangle_{\perp}}{\left(q_{z}-i \epsilon\right)^{2}} \\
= & -6 V\left(\sqrt{\mathbf{q}_{\perp}^{2}+z^{\prime 2}} ; s\right)+ \\
& +2\left(-\nabla_{\perp}^{2}-i \mathbf{q}_{\perp} \nabla_{\perp}\right) \int_{-\infty}^{z} d z^{\prime} V\left(\sqrt{\mathbf{q}_{\perp}^{2}+z^{\prime 2}} ; s\right) ; \\
W_{20}=- & 4 \int d \mathbf{q}_{1} d \mathbf{q}_{2} e^{-i\left(\mathbf{q}_{1}+\mathbf{q}_{2}\right) \mathbf{r}} V\left(\mathbf{q}_{1} ; s\right) V\left(\mathbf{q}_{2} ; s\right) \times \\
& \times \frac{3 q_{1 z} q_{2 z}+\mathbf{q}_{1 \perp} \mathbf{q}_{2 \perp}}{\left(q_{1 z}-i \varepsilon\right)\left(q_{2 z}-i \varepsilon\right)\left(q_{1 z}+q_{2 z}-i \varepsilon\right)} \\
= & -4 i\left\{3 \int_{-\infty}^{z} d z^{\prime} V^{2}\left(\sqrt{\mathbf{q}_{\perp}^{2}+z^{\prime 2}} ; s\right)\right. \\
& \left.+\left[\nabla_{\perp} \int_{-\infty}^{z^{\prime}} d z^{\prime \prime} V^{2}\left(\sqrt{\mathbf{q}_{\perp}^{2}+z^{\prime \prime 2}} ; s\right)\right]^{2}\right\}
\end{aligned}
$$

In the limit $s \rightarrow \infty$ and $(t / s) \rightarrow 0, W_{10}$ makes the main contribution, and the remaining terms are corrections. Therefore, the function $W$ can be represented by means of the expansion (19) where $W_{10}, W_{11}$ and $W_{20}$ are determined by Eqs. (24)-(26), respectively. The asymptotic behavior scattering amplitude can be written in the following form

$$
\begin{aligned}
& \left.T(s ; t)\right|_{\substack{s \rightarrow \infty \\
t-\text { fixed }}}=\frac{g}{(2 \pi)^{3}} \int d^{2} \mathbf{r}_{\perp} d z e^{i \Delta_{\perp} \mathbf{r}_{\perp}} \\
& \times V\left(\sqrt{\mathbf{r}^{2}+z^{2}} ; s\right) \\
& \quad \times \exp \left(g \frac{W_{10}}{s}\right)\left(1+g \frac{W_{11}}{s \sqrt{s}}+g^{2} \frac{W_{20}}{s^{2} \sqrt{s}}+\cdots\right) .
\end{aligned}
$$

Substituting Eqs. (24)-(26) into (27) and making calculations, at the $s \rightarrow \infty$ and t-fixed momentum transfer, we finally obtain

$$
\begin{aligned}
& \left.T(s ; t)\right|_{s \rightarrow \infty t-\text { fixed }}=-\frac{i s}{2(2 \pi)^{3}} \int d^{2} \mathbf{r}_{\perp} e^{i \mathbf{\Delta}_{\perp} \mathbf{r}_{\perp}} \\
& \quad \times\left\{e^{\left[\frac{2 i g}{s} \int_{-\infty}^{\infty} d z V\left(\sqrt{\mathbf{r}^{2}+z^{2}} ; s\right)\right]}-1\right\} \\
& \quad-\frac{6 g^{2}}{(2 \pi)^{3} s \sqrt{s}} \int d^{2} \mathbf{r}_{\perp} e^{i \Delta_{\perp} \mathbf{r}_{\perp}} \\
& \quad \times \exp \left[\frac{2 i g}{s} \int_{-\infty}^{\infty} d z V\left(\sqrt{\mathbf{r}_{\perp}^{2}+z^{2}} ; s\right)\right]
\end{aligned}
$$

$$
\begin{aligned}
& \times \int_{-\infty}^{\infty} d z V^{2}\left(\sqrt{\mathbf{r}_{\perp}^{2}+z^{2}} ; s\right) \\
& -\frac{i g}{(2 \pi)^{3} \sqrt{s}} \int d^{2} \mathbf{r}_{\perp} e^{i \boldsymbol{\Delta}_{\perp} \mathbf{r}_{\perp}} \\
& \times \int_{-\infty}^{\infty} d z\left\{\exp \left[\frac{2 i g}{s} \int_{z}^{\infty} d z^{\prime} V\left(\sqrt{\mathbf{r}_{\perp}^{2}+z^{\prime 2}} ; s\right)\right]\right. \\
& \left.-\exp \left[\frac{2 i g}{s} \int_{-\infty}^{\infty} d z^{\prime} V\left(\sqrt{\mathbf{r}_{\perp}^{2}+z^{\prime 2}} ; s\right)\right]\right\} \\
& \times\left\{\int_{z}^{\infty} d z^{\prime} \nabla_{\perp}^{2} V\left(\sqrt{\mathbf{r}_{\perp}^{2}+z^{\prime 2}} ; s\right)\right. \\
& \left.-\frac{2 i g}{s}\left[\int_{z}^{\infty} d z^{\prime} \nabla_{\perp} V\left(\sqrt{\mathbf{r}_{\perp}^{2}+z^{2}} ; s\right)\right]^{2}\right\} \\
& \left.-\frac{2 i g}{(2 \pi)^{3} s} \boldsymbol{\Delta}_{\perp}^{2} \int d^{2} \mathbf{r}_{\perp} V\left(\sqrt{\mathbf{r}_{\perp}^{2}+z^{\prime 2}} ; s\right)\right] e^{i \boldsymbol{\Delta}_{\perp} \mathbf{r}_{\perp}} \\
& \times \int_{-\infty}^{\infty} z d z V\left(\sqrt{\mathbf{r}_{\perp}^{2}+z^{2}} ; s\right) \\
& \times \exp \left[\frac{2 i g}{s} \int_{z}^{\infty} d z^{\prime} V\left(\sqrt{\mathbf{r}_{\perp}^{2}+z^{\prime 2}} ; s\right)\right]+\cdots \\
& =\left.T_{\text {Scalar }}^{(0)}(s ; t)\right|_{s \rightarrow \infty}+\left.T_{\text {Scalar }}^{(1)}(s ; t)\right|_{s \rightarrow \infty} \\
& t \text {-fixed } t \text {-fixed } \\
& \left.T_{\text {Scalar }}^{(2)}(s ; t)\right|_{s \rightarrow \infty}+\left.T_{\text {Scalar }}^{(3)}(s ; t)\right|_{s \rightarrow \infty} \\
& t \text {-fixed } t \text {-fixed }
\end{aligned}
$$

In Eq. (28) the first term describes the leading eikonal behavior of the scattering amplitude, while the remaining terms determine the corrections of relative magnitude $1 / \sqrt{s}$. Due to the smoothness of the potential $V$ at high energy $s \rightarrow \infty$ the change of the particle momentum $\Delta_{\perp}$, is relatively small. Therefore, the terms proportional to $\Delta_{\perp} V$ and $\Delta_{\perp}^{2} V$ in Eq. (28) can be neglected, now we have

$$
\begin{aligned}
& \left.T_{\text {Scalar }}^{(0)}(s ; t)\right|_{\substack{t-\text { fixed } \\
t-\infty}}=-\frac{i s}{2(2 \pi)^{3}} \int d^{2} r_{\perp} e^{i \boldsymbol{\Delta}_{\perp} \mathbf{r}_{\perp}} \\
& \times\left\{\exp \left[\frac{2 i g}{s} \int_{-\infty}^{\infty} d z V\left(\sqrt{\mathbf{r}^{2} \perp+z^{2}} ; s\right)\right]-1\right\} \\
& \left.T_{\text {Scalar }}^{(1)}(s ; t)\right|_{s \rightarrow \infty}=-\frac{6 g^{2}}{(2 \pi)^{3} s \sqrt{s}} \int d^{2} \mathbf{r}_{\perp} e^{i \boldsymbol{\Delta}_{\perp} \mathbf{r}_{\perp}} \\
& \quad \times \exp \left[\frac{2 i g}{s} \int_{-\infty}^{\infty} d z V\left(\sqrt{\mathbf{r}_{\perp}^{2}+z^{2}} ; s\right)\right] \\
& \quad \times \int_{-\infty}^{\infty} d z V^{2}\left(\sqrt{\mathbf{r}_{\perp}^{2}+z^{2}} ; s\right)
\end{aligned}
$$

As it is well known from the investigation of the scattering amplitude in the Feynman diagrammatic technique, the high energy asymptotic behavior can contain only logarithms and integral powers of $s$. A similar behavior is observed here, since the integration of Eq. (28) leads to vanishing coeffi- 
cients for half-integral powers $s$. Nevertheless, allowance for the terms that contain the half-integral powers $s$ is needed for the calculations of the next corrections in the scattering amplitude, and leads to the appearance of the so-called retardation effects, which are absent within the approximation used in the principal asymptotic terms.

For the first term from Eq. (28) in the limit of high energies $s \rightarrow \infty$ and for $t$-fixed momentum transfers, with the assumption of smooth behavior the smooth behavior of the quasi-potential as a function of the relative coordinate of two "nucleons", in the framework of quantum field theory we find the leading eikonal of the high energy scattering amplitude ${ }^{3}$

$$
\begin{aligned}
T_{\text {Scalar }}^{(0)}(s ; t) \mid \underset{s \rightarrow \infty}{t \rightarrow \text { fixed }} & -\frac{i s}{2(2 \pi)^{3}} \int d^{2} r_{\perp} e^{i \Delta_{\perp} \mathbf{r}_{\perp}} \times \\
& \times\left\{\exp \left[i \chi_{0}\left(\left|\mathbf{r}_{\perp}^{2}\right| ; s\right)\right]-1\right\} \\
\chi_{0}\left(\left|\mathbf{r}_{\perp}\right| ; s\right)= & -\frac{g^{2}}{(2 \pi)^{2} s} K_{0}\left(\mu\left|\mathbf{r}_{\perp}\right|\right)
\end{aligned}
$$

where $\left|\mathbf{r}_{\perp}\right|$ is a two-dimensional vector perpendicular to the nucleon-collision direction (the impact parameter), $K_{0}\left(\mu\left|\mathbf{r}_{\perp}\right|\right.$ ) is Mac Donald function of zeroth order, $\mu$ is a graviton mass which serves as an infrared cut-off and $\chi_{0}\left(\left|\mathbf{r}_{\perp}\right| ; s\right)$ is the eikonal phase function.

The similar leading eikonal Eq. (31) for the Lagrangian interaction $L_{i n t}=g \varphi^{2}(x) \phi(x)$ is also found by means of the functional integration[21], where eikonal phase function for exchange scalar virtual meson corresponding to a Yukawa interaction potential between two "nucleons" $V\left(\left|\mathbf{r}_{\perp}\right| ; s\right)=$ $-\left(\frac{g^{2}}{4 \pi s}\right)\left(\frac{e^{-\mu\left|\mathbf{r}_{\perp}\right|}}{\mathbf{r}_{\perp}}\right)[21]$. Use this exact form of the Yukawa as a quasi-potential and replace into Eqs. (29) and (30), for the leading eikonal amplitude and its first correction, we get (see Appendix C)

$$
\begin{aligned}
& T_{\text {Scalar }}^{(0)}(s ; t) \\
& \quad=\frac{g^{2}}{2(2 \pi)^{4} s} \cdot\left[\frac{1}{\mu^{2}-t}-\frac{g^{4}}{4(2 \pi)^{2} s^{2}} F_{1}(t)+\frac{g^{7}}{12(2 \pi)^{5} s^{4}} F_{2}(t)\right] \\
& T_{\text {Scalar }}^{(1)}(s ; t)=\frac{3 i g^{6}}{4(2 \pi)^{6} s^{3} \sqrt{s}}\left[F_{1}(t)-\frac{g^{3}}{(2 \pi)^{3} s^{2}} F_{2}(t)\right]
\end{aligned}
$$

where

$$
F_{1}(t)=\frac{1}{t \sqrt{1-4 \mu^{2} / t}} \ln \left|\frac{1-\sqrt{1-4 \mu^{2} / t}}{1+\sqrt{1-4 \mu^{2} / t}}\right|,
$$

\footnotetext{
3 The amplitude $T$ is normalized in the c.m.s by the relation $\frac{d \sigma}{d \Omega}=$ $\frac{|T(s, t)|^{2}}{64 \pi^{2} s}, \sigma_{t}=\frac{1}{2 p \sqrt{s}} \operatorname{Im} T(s, t=0)$.
}

and

$$
F_{2}(t)=\int_{0}^{1} d y \frac{1}{\left(t y+\mu^{2}\right)(y-1)} \ln \left|\frac{\mu^{2}}{y\left(t y+\mu^{2}-t\right)}\right|
$$

The similar calculations can be applied for other exchanges with different spins. In the case of the vector model $L_{i n t}=$ $-g \varphi^{\star} i \partial_{\sigma} \varphi A^{\sigma}+g^{2} A_{\sigma \sigma} A^{\sigma} \varphi \varphi^{\star} \varphi$ the quasi-potential is independent of energy $V\left(\left|\mathbf{r}_{\perp}\right|\right)=-\left(g^{2} / 4 \pi\right)\left(e^{-\mu\left|\mathbf{r}_{\perp}\right|} /\left|\mathbf{r}_{\perp}\right|\right)$, we get

$$
\begin{aligned}
& T_{\text {Vector }}^{(0)}(s, t) \\
& \quad=\frac{g^{2}}{2(2 \pi)^{4}} \cdot\left[\frac{1}{\mu^{2}-t}-\frac{g^{4}}{4(2 \pi)^{2} s} F_{1}(t)+\frac{g^{7}}{12(2 \pi)^{5} s^{2}} F_{2}(t)\right] \\
& T_{\text {Vector }}^{(1)}(s, t)=\frac{3 i g^{6}}{4(2 \pi)^{6} s \sqrt{s}}\left[F_{1}(t)-\frac{g^{3}}{(2 \pi)^{3} s} F_{2}(t)\right]
\end{aligned}
$$

In the case of tensor model ${ }^{4}$, the quasi- potential increases with energy

$V\left(\left|\mathbf{r}_{\perp}\right| ; s\right)=\left(\kappa^{2} s / 4 \pi\right)\left(e^{-\mu\left|\mathbf{r}_{\perp}\right|} /\left|\mathbf{r}_{\perp}\right|\right)$,

we have

$$
\begin{aligned}
& T_{\text {Tensor }}^{(0)}(s, t) \\
& \quad=\frac{\kappa^{2} s}{(2 \pi)^{4}} \cdot\left[\frac{1}{\mu^{2}-t}-\frac{\kappa^{4}}{2(2 \pi)^{2}} F_{1}(t)+\frac{\kappa^{7}}{3(2 \pi)^{5}} F_{2}(t)\right] \\
& T_{\text {Tensor }}^{(1)}(s, t)=\frac{3 i \kappa^{6} \sqrt{s}}{(2 \pi)^{6}}\left[F_{1}(t)-\frac{2 \kappa^{3}}{(2 \pi)^{3}} F_{2}(t)\right]
\end{aligned}
$$

Comparison of these above potentials has made it possible to draw the following conclusions: in the model with the scalar exchange, the total cross section $\sigma_{t}$ decreases as $\frac{1}{s}$, and only the Born term predominates in the entire eikonal equation; the vector model leads to a total cross section $\sigma_{t}$ approaching a constant value as $s \rightarrow \infty, \frac{t}{s} \rightarrow 0$. In both cases, the eikonal phases are purely real and consequently the influence of inelastic scattering is disregarded in this

\footnotetext{
4 The model of interaction of a scalar "nucleons" with a gravitational field in the linear approximation to $h^{\mu \nu}(x) L(x)=L_{0, \varphi}(x)+$ $L_{0, \text { grav. }}(x)+L_{\text {int }}(x)$, where

$L_{0}(x)=\frac{1}{2}\left[\partial^{\mu} \varphi(x) \partial_{\mu} \varphi(x)-m^{2} \varphi^{2}(x)\right]$,

$L_{i n t}(x)=-\frac{\kappa}{2} h^{\mu \nu}(x) T_{\mu v}(x)$,

$T_{\mu \nu}(x)=\partial_{\mu} \varphi(x) \partial_{\nu} \varphi(x)-\frac{1}{2} \eta_{\mu \nu}\left[\partial^{\sigma} \varphi(x) \partial_{\sigma} \varphi(x)-m^{2} \varphi^{2}(x)\right]$,

$T_{\mu \nu}(x)$ is the energy momentum tensor of the scalar field. The coupling constant $\kappa$ is related to Newton's constant of gravitation $G$ by $\kappa^{2}=$ $32 \pi G=32 \pi l_{P L}^{2}, l_{P L}=1,6.10^{-33} \mathrm{~cm}$ is the Planck length.
} 
approximation, $\sigma^{i n}=0$. In the case of graviton exchange the Froissart limit is violated. A similar result is also obtained in Ref. [18] with the eikonal series for reggeized graviton exchange.

In the framework of the quasi-potential approach and the modified perturbation theory a systematic scheme of finding the leading eikonal scattering amplitudes and its corrections are developed and constructed in quantum field theory including the linearized gravity. The first correction to leading eikonal amplitude is found.

\section{The one loop approximation contribution to high energy scattering}

The low energy effective theory of quantized gravity is currently our most successful attempt at unifying general relativity and quantum mechanics [51-70]. Therefore, we attempt to extend the above approach to calculating the high energy scattering amplitude of two "nucleons" for the graviton exchange based on the Newtonian potential with lowenergy leading one loop gravitational corrections of two large non-relativistic masses [53]

$$
\begin{aligned}
& V_{\text {Newton }}(r) \\
& \quad=-G \frac{m_{1} m_{2}}{r}\left[1+3 \frac{G\left(m_{1}+m_{2}\right)}{c^{2} r}+\frac{41}{10 \pi} \frac{G \hbar}{c^{3} r^{2}}\right]
\end{aligned}
$$

It is important to note that classical post-Newtonian term in expression (40) corresponds to the lowest-order scattering potential and agrees with Eq. (2.5) of Iwasaki [55]. The correct result for the quantum corrections first published [53] and later confirmed in [62].

When substituting the Newtonian potential (40) into Eqs. (29) and (30), the vertex factor for graviton exchange between "nucleons" should be added by $V_{\text {Newton }}$ with $s V_{\text {Newton }}[21$, 62], the definition of the non-relativistic potential (see Eq. (D.7) in Appendix D) and graviton still has a mass $\mu$ and perform some calculations for the leading eikonal behavior and the first correction of the scattering amplitude (see Eqs. (E.3) and (E.7) in Appendix E) we find the following expressions

$$
\begin{aligned}
& T_{\text {graviton }}^{(0)}(s, t) \\
& =\frac{\kappa^{2} s}{(4 \pi)^{4}}\left(\frac{1}{\mu^{2}-t}-\frac{\kappa^{3}}{2 \cdot(32 \pi)^{2}} F_{1}(t)+\frac{2 \kappa^{6}}{3 .(16 \pi)^{5}} F_{2}(t)\right) \\
& \quad-\frac{6\left(m_{1}+m_{2}\right)}{(32 \pi)^{3} c^{2}} \frac{\kappa^{5} s}{\sqrt{\mu^{2}-t}}+\frac{41 \kappa^{5} s \hbar}{80 .(4 \pi)^{5} c^{3}} F_{2}(t)
\end{aligned}
$$

and

$$
\begin{gathered}
T_{\text {graviton }}^{(1)}(s, t)=\frac{3 i \kappa^{6} \sqrt{s}}{(8 \pi)^{6}}\left[F_{1}(t)-\frac{2 \kappa^{4}}{(8 \pi)^{3}} F_{2}(t)\right] \\
+\frac{9\left(m_{1}+m_{2}\right)}{4(8 \pi)^{3} c^{2}} \frac{\kappa^{6} \sqrt{s}}{\sqrt{\mu^{2}-t}}+\frac{123 \kappa^{6} \hbar \sqrt{s}}{10(4 \pi)^{5} c^{3}} F_{2}(t)
\end{gathered}
$$

From the Eqs. (41) and (42) above, we see that the leading eikonal term and the first correction term of scattering amplitude have the same structure including three small terms: (i) The first term is the scattering amplitude by exchanging gravitons, which in its non-relativistic limit will be the Newton potential; (ii) The second term is the relativistic correction for the scattering amplitude [the term containing $\left.\left(m_{1}+m_{2}\right)\right]$. This term corresponds to the non-analytic contribution because of exchanging gravitons. The relativistic correction term is explained as the "zitterbewegung" fluctuation when the distance between two interacting particles is shifted by one Compton wavelength [51-53]; (iii) The last term (the term proportional to $\hbar$ ) is quantum correction, obtained from the contribution of the one loop diagram in the high energy scattering process.

The quantum correction term found in the linear gravitational field corresponds to the local interaction that is related to the analytical properties of the scattering amplitude. The Newtonian potential and its quantum corrections are related to the non-locality of the quasi-potential; non-analytic terms are also related to the non-locality of the Newtonian potential.

The lowest order approximation of the modified perturbation theory is the leading eikonal scattering amplitude with phase function defined by the smooth local Yukawa quasipotential in linearized quantum gravity. Using the Yukawa quasi-potential corresponding to virtual graviton between two "nucleons" we obtain the first correction to leading eikonal amplitude.

In the effective quantum gravity, the Newtonian quasipotential, which contains the relativistic, and quantum corrections, we found the one-loop leading eikonal scattering amplitude and its first correction to the leading amplitude [the last terms in Eqs. (41) and (42)]. The difference here of the non-analytic and nonlocal contributions, connected with nonlocal quasi-potential were discussed and included in the calculation of the behavior scattering amplitude.

\section{Conclusion}

In the framework of the modified perturbation theory and the quasi-potential equation, a systematic scheme of finding the leading eikonal scattering amplitudes and its corrections in the one-loop gravitational approximation in quantum gravity are developed and constructed. The first non-leading corrections to leading eikonal amplitude are found. 
In the linearized gravitational theory, the interaction between of scattered "nucleons" by exchanging graviton, which corresponds to the smooth quasi-potential of the Yukawa type. The leading eikonal scattering amplitude and its first correction also found.

In the framework of effective field theory, we obtained the expression for the scattering amplitude in Newtonian potential taking into account the contribution of relativistic and quantum corrections from the one-loop diagram. The difference with the above case includes: (i) relativistic correction terms calculated from non-analytical contributions and explained as a result of "zitterbewegung" fluctuations when the distance between particles is shifted one Compton wavelength; (ii) quantum correction terms related to Planck's constant $\hbar$ was also found.

The contributions to the high energy scattering amplitude are divided into analytic contributions related to the locality and non-analytic contributions associated with the nonlocality. This division is associated with two ways of describing particles in quantum mechanics and relativistic quantum mechanics in that the particle has mass $m$. If the particle has mass, it is not possible to the localization of the particle in a volume with linear dimensions less than the Compton wavelength of the corresponding particle. For ultra-relativistic particles such as light quanta for which $(m=0, v=c)$-the concept of the coordinates of the particle in the usual sense of the word is completely meaningless [70].

The new results in this paper are that we have incorporated relativistic effects and quantum effects in gravitational scattering to clarify part of the connection between the effective quantum field theory of general relativity and relativistic quantum mechanics.

Acknowledgements We are grateful to Profs. B. M. Barbashov,V. A. Efremov, A. B. Arbuzov, V. R. Goloskokov, V. P. Gerdt, A.J. Silenko for valuable discussions. NSH is also indebted to Prof. G. Veneziano for suggesting this problem and his encouragement, and Prof. H. Fried for useful remarks related to the modified perturbation theory. This work was supported in part by the joint Institute Nuclear Research (JINR, Dubna), the Hanoi University of Science and funded by Vietnam National Foundation for Science and Technology Development (NAFOSTED) under Grant Number 103.01-2018.42. DTH is supported in part by the project 911 of Hanoi University of Science - VNU HN.

Data Availability Statement This manuscript has no associated data or the data will not be deposited. [Authors' comment: This is a theoretical study and no experimental data has been listed.]

Open Access This article is distributed under the terms of the Creative Commons Attribution 4.0 International License (http://creativecomm ons.org/licenses/by/4.0/), which permits unrestricted use, distribution, and reproduction in any medium, provided you give appropriate credit to the original author(s) and the source, provide a link to the Creative Commons license, and indicate if changes were made.

Funded by SCOAP ${ }^{3}$.
Appendix A: The smoothness of the local quasi-potential

Here we demonstrate that the eikonal representation is a consequence of the assumption of the non-singular character of hadron interactions at high energies. We shall use a quasipotential equation for the wave function of two particles in the coordinate representation which has the form of a nonlocal differential equation

$$
\left(E^{2}-m^{2}+\nabla^{2}\right) \psi(\mathbf{r})=\frac{1}{\sqrt{m^{2}-\nabla^{2}}} V(\mathbf{r}, s) \psi(\mathbf{r})
$$

where $\mathbf{r}$ is the vector of the relative coordinate of two "nucleons". Under the condition of non-singular or smooth behavior quasi-potential $V(\mathbf{r}, s)$, the non-localized differential equation Eq. (A.1) takes an effectively local form in the highenergy limit. Actually, let us look for a solution of Eq. (A.1) of the form

$\psi(\mathbf{r})=e^{i p z} \varphi(\mathbf{r})$

where $\varphi(\mathbf{r})$ is expected to be a slowly varying functions compared to $\exp (i p z)$ and $E=\sqrt{\mathbf{p}^{2}+m^{2}}$.

It can easily be shown that on a space of slowly varying functions in the high-energy limit $p \rightarrow \infty$

$e^{-i p z} \frac{1}{\sqrt{m^{2}-\nabla^{2}}} e^{i p z} \rightarrow p-i \nabla_{z}+O\left(\frac{1}{p}\right)$

or

$e^{-i p z}\left(\sqrt{m^{2}-\nabla^{2}}\right) e^{i p z} \rightarrow \frac{1}{p}+O\left(\frac{1}{p^{2}}\right)$

Thus the function $\varphi(\mathbf{r})$ obeys the equation

$-2 i p \frac{\partial \varphi(\mathbf{r})}{\partial z}=\frac{1}{p} V(s, r) \varphi(\mathbf{r})$

which coincides with the one which follows from the local Klein-Gordon equation with effective potential $\frac{1}{p} V(\mathbf{r}, s)$. As a result we have the eikonal representation of elastic scattering amplitude with eikonal phase function $\chi_{0}\left(\left|\mathbf{r}_{\perp}\right|, s\right)$ in Eq. (31). It is important note that the nonlocal potential differs from the local by terms of order $O\left(\frac{1}{p}\right)$ in the high energy limit $p \rightarrow \infty$. It should be noted, shifting from describing non-relativistic quantum theory to relativistic descriptions requires changing the concept of the coordinates of individual particles according to mass values. If the particle has mass, it is not possible to locate a particle in space with a length smaller than the Compton wavelength of the corresponding particle. For limited relativistic particles - light quanta - the concept of the particle's coordinates in the usual sense is completely absent [70]. 


\section{Appendix B: Relationship between the operator and Feynman path methods}

What actual physical picture may correspond to our result given by Eq. (28)? To answer this question, we establish the relationship between the operator and Feynman path methods, which treats the quasi-potential equation Eq. (10) in the language of functional integrals. The solution of this equation can be written in the symbolic form

$$
\begin{aligned}
\exp (W) & =\frac{1}{1-g K\left[(-i \nabla-\mathbf{k})^{2}\right] V(\mathbf{r})} \times 1 \\
& =-i \int_{0}^{\infty} d \tau e^{i \tau(1+i \varepsilon)} \exp \left\{-i \tau g K\left[(-i \nabla-\mathbf{k})^{2}\right] V(\mathbf{r})\right\} \times 1
\end{aligned}
$$

In accordance with the Feynman parametrization, we introduce an ordering index $\eta$ and write Eq. (B.1) in the form

$$
\begin{aligned}
\exp (W)= & -i \int_{0}^{\infty} d \tau e^{i \tau(1+i \varepsilon)} \\
& \times \exp \left\{-i g \int_{0}^{\infty} d \eta K\left[\left(-i \nabla_{\eta+\varepsilon}-\mathbf{k}\right)^{2}\right] V\left(\mathbf{r}_{\eta}\right)\right\} \times 1
\end{aligned}
$$

Using Feynman transformation

$$
\begin{aligned}
F[P(\eta)]= & \int D \mathbf{p} \int_{x(0)=0} \frac{D \mathbf{x}}{(2 \pi)^{3}} \\
& \times \exp \left\{i \int_{0}^{\tau} d \eta \overrightarrow{\dot{r}}[\mathbf{p}(\eta)-P(\eta)]\right\} F[\mathbf{p}(\eta)]
\end{aligned}
$$

We write the solution of Eq. (6) in the form of the functional integral

$$
\begin{aligned}
\exp (W)= & -i \int_{0}^{\infty} d \tau e^{i \tau(1+i \varepsilon)} \int D \mathbf{p} \int_{x(0)=0} \frac{D \mathbf{x}}{(2 \pi)^{3}} \\
& \times \exp \left\{i \int_{0}^{\tau} d \eta \vec{x}(\eta)[\mathbf{p}(\eta)-P(\eta)]\right\} G(\mathbf{x}, \mathbf{p} ; \tau) \times 1
\end{aligned}
$$

In Eq. (B.4) we enter the function $G$

$G(\mathbf{x}, \mathbf{p} ; \tau) \exp \left\{-i \int_{0}^{\tau} d \eta \overrightarrow{\dot{x}}(\eta) \nabla_{\eta+\varepsilon}\right\}$

$$
\times \exp \left\{-i g \int_{0}^{\tau} d \eta K\left[(\mathbf{p}(\eta)-\mathbf{k})^{2}\right] V\left(\mathbf{r}_{\eta}\right)\right\}
$$

Which satisfies the equation

$$
\begin{aligned}
\frac{d G}{d \tau} & =\left\{-i g K\left[(\mathbf{p}(\tau)-\mathbf{k})^{2}\right] V(\mathbf{r}-\vec{x}(\tau-\varepsilon)) \nabla\right\} G ; \\
G(\tau=0) & =1
\end{aligned}
$$

Finding from Eq. (B.6) the operator function $G$ and substituting it into Eq. (B.4) for $W$ we obtained the following final expression

$$
\begin{aligned}
\exp (W)= & -i \int_{o}^{\infty} d \tau e^{i \tau(1+i \varepsilon)} \int D \mathbf{p} \int_{x(0)=0} \frac{D \mathbf{x}}{(2 \pi)^{3}} \\
& \times \exp \left\{i \int_{0}^{\tau} d \eta \overrightarrow{\dot{x}}(\eta) \mathbf{p}(\eta)\right\} \exp (g \Pi)
\end{aligned}
$$

where

$$
\begin{aligned}
\Pi= & -i \int_{0}^{\tau} d \eta K\left[(\mathbf{p}(\eta)-\mathbf{k})^{2}\right] \\
& \times V\left[\mathbf{r}-\int_{0}^{\tau} d \xi \mathbf{x}(\xi) \vartheta(\xi-\eta+\varepsilon)\right] \\
\Pi^{2}= & -i \int_{0}^{\tau_{1}} \int_{0}^{\tau_{2}} d \eta_{1} d \eta_{2} K\left[\left(\mathbf{p}\left(\eta_{1}\right)-\mathbf{k}\right)^{2}\right] K\left[\left(\mathbf{p}\left(\eta_{2}\right)-\mathbf{k}\right)^{2}\right] \\
& \times V\left[\mathbf{r}_{1}-\int_{0}^{\tau_{1}} d \xi \mathbf{x}(\xi) \vartheta(\xi-\eta+\varepsilon)\right] \\
& \times V\left[\mathbf{r}_{2}-\int_{0}^{\tau_{2}} d \xi \mathbf{x}(\xi) \vartheta(\xi-\eta+\varepsilon)\right]
\end{aligned}
$$

Writing out the expansion

$\overline{\exp (g \Pi)}=\exp (g \bar{\Pi}) \sum_{n=0}^{\infty} \frac{g^{n}}{n !} \overline{(\Pi-\bar{\Pi})^{n}}$

in which the sign of averaging denoted integration with respect to $\tau, \mathbf{x}(\eta)$ and $\mathbf{p}(\eta)$ with the corresponding measure (see, for example Eq. (B.7)), and performing the calculations, we find

$$
\begin{aligned}
& W_{1}=\bar{\Pi}, \quad W_{2}=\frac{1}{2 !}\left(\overline{\Pi^{2}}-\bar{\Pi}^{2}\right), \\
& W_{3}=\frac{1}{3 !}\left[\overline{\Pi^{3}}-\bar{\Pi}^{3}-3 \bar{\Pi}\left(\overline{\Pi^{2}}-\bar{\Pi}^{2}\right)\right] \cdots \text { etc }
\end{aligned}
$$


the Eqs. (B.11) and (13), (14), (15) are identical.

$$
\begin{aligned}
& W_{1}(\mathbf{r} ; \mathbf{k} ; s)=\bar{\Pi}=-i \int_{0}^{\infty} d \eta K\left[(\mathbf{p}(\eta)-\mathbf{k})^{2}\right] \\
& \quad \times \exp \left[-\int_{0}^{\tau} d \xi \mathbf{x}(\xi) \vartheta(\xi-\eta+\varepsilon)\right] V(\mathbf{r}) \\
&=\int d \mathbf{q} e^{-i \mathbf{q r}} K\left[(\mathbf{q}+\mathbf{k})^{2}\right] V(\mathbf{q} ; s), \\
& \overline{\Pi^{2}}= K\left[\left(\nabla_{\mathbf{r}_{1}}+\nabla_{\mathbf{r}_{2}}+\mathbf{k}\right)^{2}\right] K\left[\left(\nabla_{\mathbf{r}_{1}}+\mathbf{k}\right)^{2}\right] \\
& \times K\left[\left(\nabla_{\mathbf{r}_{2}}+\mathbf{k}\right)^{2}\right] V\left(\mathbf{r}_{1} ; s\right) V\left(\mathbf{r}_{2} ; s\right) \\
&= \int d \mathbf{q}_{1} \int d \mathbf{q}_{2} e^{-i\left(\mathbf{q}_{1}+\mathbf{q}_{2}\right) \mathbf{r}} K\left[\left(\mathbf{k}+\mathbf{q}_{1}+\mathbf{q}_{2}\right)^{2}\right] \\
& \times\left\{K\left[\left(\mathbf{k}+\mathbf{q}_{1}\right)^{2} ; s\right]+K\left[\left(\mathbf{k}+\mathbf{q}_{2}\right)^{2} ; s\right]\right\} V\left(\mathbf{r}_{1} ; s\right) V\left(\mathbf{r}_{2} ; s\right)
\end{aligned}
$$

$$
\begin{aligned}
& W_{2}(\mathbf{r} ; \mathbf{k} ; s)=-\frac{W_{1}^{2}(\mathbf{r} ; \mathbf{k} ; s)}{2 !} \\
& \quad+\frac{1}{2} \int d \mathbf{q}_{1} \int d \mathbf{q}_{2} V\left(\mathbf{q}_{1} ; s\right) V\left(\mathbf{q}_{2} ; s\right) \\
& \quad \times\left\{K\left[\left(\mathbf{q}_{1}+\mathbf{k}\right)^{2} ; s\right]+K\left[\left(\mathbf{q}_{2}+\mathbf{k}\right)^{2} ; s\right]\right\} e^{-i\left(\mathbf{q}_{1}+\mathbf{q}_{2}\right) \mathbf{r}}
\end{aligned}
$$$$
W_{3}(\mathbf{r} ; \mathbf{k} ; s)=-\frac{W_{1}^{2}(\mathbf{r} ; \mathbf{k} ; s)}{3 !}
$$$$
+\int d \mathbf{q}_{1} d \mathbf{q}_{2} d \mathbf{q}_{3} V\left(\mathbf{q}_{1} ; s\right) V\left(\mathbf{q}_{2} ; s\right) V\left(\mathbf{q}_{3} ; s\right)
$$$$
\times K\left[\left(\mathbf{q}_{1}+\mathbf{k}\right)^{2} ; s\right] K\left[\left(\mathbf{q}_{2}+\mathbf{k}\right)^{2} ; s\right]
$$$$
\times K\left[\left(\mathbf{q}_{3}+\mathbf{k}\right)^{2} ; s\right] e^{-i\left(\mathbf{q}_{1}+\mathbf{q}_{2}+\mathbf{q}_{3}\right) \mathbf{r}}
$$

Restricting ourselves in the expansion (B.11) to the first term, we obtain the approximate Eq. (29) for the scattering amplitude, which corresponds to the allowance for the particle Feynman paths. These paths can be considered as a classical paths and coincide in the case of the scattering of high energy particles through small angles to straight-line paths trajectories.

\section{Appendix C: Some integrals used in this paper}

Firstly, we consider the integral

$I_{1}=\int_{-\infty}^{\infty} d z V\left(\sqrt{r_{\perp}^{2}+z^{2}}, s\right)=-\frac{g^{2}}{4 \pi s} \int_{-\infty}^{\infty} d z \frac{e^{-\mu \sqrt{r_{\perp}^{2}+z^{2}}}}{\sqrt{r_{\perp}^{2}+z^{2}}}$

here $V(r ; s)=-\left(g^{2} B i g / 4 \pi s\right)\left(e^{-\mu r} / r\right)$ is the Yukawa interaction potential between two "nucleons".
Perform Fourier transformation

$$
\begin{aligned}
I_{1} & =-\frac{g^{2}}{4 \pi s} \int_{-\infty}^{\infty} d z\left(\frac{1}{(2 \pi)^{3}} \int d^{3} k \frac{e^{-\mathbf{k r}}}{k^{2}+\mu^{2}}\right) \\
& =-\frac{g^{2}}{2(2 \pi)^{3} s} \int d^{2} k_{\perp} \frac{e^{-i k_{\perp} r_{\perp}}}{k_{\perp}^{2}+\mu^{2}}=-\frac{g^{2}}{2(2 \pi)^{2} s} K_{0}\left(\mu\left|\mathbf{r}_{\perp}\right|\right)
\end{aligned}
$$

with $K_{0}\left(\mu\left|\mathbf{r}_{\perp}\right|=\frac{1}{2 \pi} \int d^{2} k_{\perp} \frac{e^{-i k_{\perp} r_{\perp}}}{k_{\perp}^{2}+\mu^{2}}\right.$ is the Mac Donald of zeroth order.

The integral

$$
\begin{aligned}
I_{2} & =\int d^{2}\left|\mathbf{r}_{\perp}\right| e^{i \Delta_{\perp} \mathbf{r}_{\perp}} K_{0}\left(\mu\left|\mathbf{r}_{\perp}\right|\right) \\
& =2 \pi \int d\left|\mathbf{r}_{\perp}\right|\left|\mathbf{r}_{\perp}\right| J_{0}\left(\boldsymbol{\Delta}_{\perp} \mathbf{r}_{\perp}\right)=\frac{2 \pi}{\mu^{2}+\Delta_{\perp}^{2}}=\frac{2 \pi}{\mu^{2}-t} .
\end{aligned}
$$

The integral

$$
\begin{aligned}
I_{3} & =\int d^{2}\left|\mathbf{r}_{\perp}\right| e^{i \Delta_{\perp}\left|\mathbf{r}_{\perp}\right|} K_{0}^{2}\left(\mu\left|\mathbf{r}_{\perp}\right|\right) \\
& =\int d^{2} q \frac{1}{q^{2}+\mu^{2}} \frac{1}{\left(q+\Delta_{\perp}\right)^{2}+\mu^{2}}
\end{aligned}
$$

here, the result of the integral that obtained from calculating $I_{2}$ have been used.

Using method of Feynman parameter integral $\frac{1}{a b}=$ $\int_{0}^{1} d x \frac{1}{[a x+b(1-x)]^{2}}$, we obtain

$$
I_{3}=(-i \pi) \int_{0}^{1} d x \frac{1}{\left[\mu^{2}-t x(1-x)\right]}=(-i \pi) F_{1}(t)
$$

with the $F_{1}(t)$-function is determined by the expression

$F_{1}(t)=\frac{1}{t \sqrt{1-4 \mu^{2} / t}} \ln \left|\frac{1-\sqrt{1-4 \mu^{2} / t}}{1+\sqrt{1-4 \mu^{2} / t}}\right|$.

By the same method, the following integral is also calculated

$$
\begin{aligned}
I_{4}= & \int d^{2}\left|\mathbf{r}_{\perp}\right| e^{i \Delta_{\perp}\left|\mathbf{r}_{\perp}\right|} K_{0}^{3}\left(\mu\left|\mathbf{r}_{\perp}\right|\right) \\
I_{4}= & \frac{1}{(2 \pi)^{2}} \int d^{2} q_{1} \int d^{2} q_{2} \\
& \times \frac{1}{q_{1}^{2}+\mu^{2}} \frac{1}{q_{2}^{2}+\mu^{2}} \frac{1}{\left(q_{1}+q_{2}+\Delta_{\perp}\right)^{2}+\mu^{2}}=-\frac{1}{2} F_{2}(t)
\end{aligned}
$$

with the $F_{2}(t)$-function is determined by the expression

$$
F_{2}(t)=\int_{0}^{1} d y \frac{1}{\left(t y+\mu^{2}\right)(y-1)} \ln \left|\frac{\mu^{2}}{y\left(t y+\mu^{2}-t\right)}\right| \text {. }
$$




\section{Appendix D: Scattering matrix and potential [53]}

In this Appendix we list some of the facts that obtained in [53].

1. The scattering amplitude for two scalar massive particles as a function of the momentum transfer $q^{2}=\left(p-p^{\prime}\right)^{2}$ in the mixed gravity-scalar theory can be expanded as

$$
\begin{aligned}
T & \sim\left[A+B q^{2}+\cdots\right. \\
& \left.+\alpha \kappa^{4} \frac{1}{q^{2}}+\beta_{1} \kappa^{4} \ln \left(-q^{2}\right)+\beta_{2} \kappa^{4} \frac{m}{\sqrt{-q^{2}}}+\cdots\right]
\end{aligned}
$$

where the coefficients $A, B \cdots$ and $\alpha, \beta_{1}, \beta_{2}$ depend on the particle masses $m_{1}, m_{2}$. The terms with $A, B \cdots$ in Eq. (D.1) are analytical in $q^{2}$ and correspond to local interactions and the other terms with $\alpha, \beta_{1}, \beta_{2}$ correspond to the non-local, long-ranged interactions, described by the nonanalytic potential.

The space parts of the non-analytical terms are performed Fourier transformation

$$
\begin{gathered}
\int \frac{d^{3} q}{(2 \pi)^{3}} e^{-i \mathbf{q r}} \frac{1}{|\mathbf{q}|^{2}}=\frac{1}{4 \pi r} ; \int \frac{d^{3} q}{(2 \pi)^{3}} e^{-i \mathbf{q r} \mathbf{r} \frac{1}{|\mathbf{q}|}=\frac{1}{2 \pi^{2} r^{2}}} \\
\int \frac{d^{3} q}{(2 \pi)^{3}} e^{-i \mathbf{q r}} \ln \left(\frac{|\mathbf{q}|^{2}}{\mu^{2}}\right)=-\frac{1}{2 \pi r^{3}} .
\end{gathered}
$$

so clearly these terms will contribute to the long range corrections. It should be noted that such nonanalytic pieces of the scattering amplitude are essential to the unitarity of the $S$ matrix.

2. In the quantization of General Relativity the definition of a potential is certainly not obvious. One can choose between several definitions of the potential depending on, e.g., the physical situation, how to define the energy of the fields, the diagrams included etc. The choice of potential which includes all 1-loop diagrams $[60,64,65]$ seems to be the simples, gauge invariant definition of the potential.

We will calculate the non-relativistic potential using the full amplitude. Here we simply relate the expectation value for the $S$ matrix to the Fourier transformation of the potential $\tilde{V}(\mathbf{q})$ in the

$$
<p_{1}^{\prime}, p_{2}^{\prime}|S| p_{1}, p_{2}>=-i \tilde{V}(\mathbf{q})(2 \pi) \delta\left(E-E^{\prime}\right)
$$

where $p, p^{\prime}$ is the incoming, outgoing four-momentum, respectively, and $\left(E-E^{\prime}\right)$ is the energy difference between the incoming and outgoing states. Comparing this to the definition of the invariant matrix element $M$ we get from diagrams

$$
<p_{1}^{\prime}, p_{2}^{\prime}|S| p_{1}, p_{2}>=(2 \pi)^{4} \delta^{(4)}\left(p_{1}+p_{2}-p_{1}^{\prime}-p_{2}^{\prime}\right)(i M)
$$

We see that we have divided the above equation with $\left(2 m_{1} 2 m_{2}\right)$ to obtain the non-relativistic limit $(q=(0, \mathbf{q}))$

$$
\tilde{V}(\mathbf{q})=-\frac{1}{2 m_{1}} \frac{1}{2 m_{2}} M
$$

so that

$$
V(\mathbf{r})=-\frac{1}{2 m_{1}} \frac{1}{2 m_{2}} \int \frac{d q^{3}}{(2 \pi)^{3}} e^{i \mathbf{q} \mathbf{r}} M(\mathbf{q})
$$

This will be our definition of the non-relativistic potential generated by the considered non-analytic parts, where $\mathrm{M}$ is the non-analytical part of the amplitude of the scattering process to a given loop order.

3. We evaluate all diagrams which contribute to the one loop scattering amplitude. Finally, we find the leading corrections to the nonrelativistic gravitational potential.

$$
\begin{aligned}
& V_{\text {Newton }}(r)= \\
& \quad-G \frac{m_{1} m_{2}}{r}\left[1+3 \frac{G\left(m_{1}+m_{2}\right)}{c^{2} r}+\frac{41}{10 \pi} \frac{G \hbar}{c^{3} r^{2}}\right],
\end{aligned}
$$

which includes the lowest-order relativistic correction, and the lowest-order quantum correction (also relativistic).

\section{Appendix E: The Newtonian potential and scattering amplitude}

The Newtonian potential with low-energy leading one-loop gravitational corrections Eq. (40) can be rewritten as

$V_{\text {Newton }}(r, s)=C_{1} \frac{\kappa^{2} s}{r}+C_{2} \frac{\kappa^{4} s}{r^{2}}+C_{3} \frac{\kappa^{4} s}{r^{3}}$,

where $C_{1}=\frac{1}{4 .(32 \pi)}, C_{2}=\frac{3\left(m_{1}+m_{2}\right)}{4 c^{2} \cdot(32 \pi)^{2}}, C_{3}=\frac{41 \hbar}{40 \pi c^{3} \cdot(32 \pi)^{2}}$.

Substituting Eq. (E.1) into Eq. (29) in case of graviton exchange, we obtain

$$
\begin{aligned}
& T_{\text {graviton }}^{(0)}(s, t) \approx \\
& \frac{\kappa}{(2 \pi)^{3}} \int d^{2} \mathbf{r}_{\perp} e^{i \boldsymbol{\Delta}_{\perp} \mathbf{r}_{\perp}}\left(C_{1} \kappa^{2} s \int_{-\infty}^{\infty} \frac{1}{\sqrt{r_{\perp}^{2}+z^{2}}} d z\right. \\
& \left.\quad+C_{2} \kappa^{4} s \int_{-\infty}^{\infty} \frac{1}{r_{\perp}^{2}+z^{2}} d z+C_{3} \kappa^{4} s \int_{-\infty}^{\infty} \frac{1}{\left(r_{\perp}^{2}+z^{2}\right)^{3 / 2}} d z\right) \\
& +\frac{i \kappa^{2}}{(2 \pi)^{3} s} \int d^{2} \mathbf{r}_{\perp} e^{i \Delta_{\perp} \mathbf{r}_{\perp}}\left(C_{1} \kappa^{2} s \int_{-\infty}^{\infty} \frac{1}{\sqrt{r_{\perp}^{2}+z^{2}}} d z\right)^{2} \\
& \quad-\frac{2 \kappa^{3}}{3(2 \pi)^{3} s^{2}} \int d^{2} \mathbf{r}_{\perp} e^{i \Delta_{\perp} \mathbf{r}_{\perp}}\left(C_{1} \kappa^{2} s \int_{-\infty}^{\infty} \frac{1}{\sqrt{r_{\perp}^{2}+z^{2}}} d z\right)^{3}
\end{aligned}
$$


Using the integrals $I_{2}, I_{3}, I_{4}$ in the Appendix $\mathrm{C}$ and the explicit coefficients $C_{1}, C_{2}, C_{3}$ above to Eq. (E.2), we receive the leading term of the scattering amplitude

$$
\begin{aligned}
& T_{\text {graviton }}^{(0)}(s, t) \\
& =\frac{\kappa^{2} s}{(4 \pi)^{4}}\left(\frac{1}{\mu^{2}-t}-\frac{\kappa^{4}}{2 .(32 \pi)^{2}} F_{1}(t)+\frac{2 \kappa^{7}}{3 .(16 \pi)^{5}} F_{2}(t)\right) \\
& \quad-\frac{6\left(m_{1}+m_{2}\right)}{(32 \pi)^{3} c^{2}} \frac{\kappa^{5} s}{\sqrt{\mu^{2}-t}}+\frac{41 \kappa^{5} s \hbar}{80 .(4 \pi)^{5} c^{3}} F_{2}(t) .
\end{aligned}
$$

By the same way, Eq. (30) is now available

$$
\begin{aligned}
& \left.T_{\text {graviton }}^{(1)}(s, t)\right|_{s \rightarrow \infty ; t-\text { fixed }} \\
& \approx-\frac{6 \kappa^{2}}{(2 \pi)^{3} s \sqrt{s}} \cdot \int d^{2} \mathbf{r}_{\perp} e^{i \mathbf{\Delta}_{\perp} \mathbf{r}_{\perp}} \times\left\{\int _ { - \infty } ^ { \infty } d z \left[\left(C_{1} \frac{\kappa^{2} s}{\sqrt{r_{\perp}^{2}+z^{2}}}\right)^{2}\right.\right. \\
& \left.\left.+2 C_{1} C_{2} \frac{\kappa^{6} s^{2}}{\sqrt{\left(r_{\perp}^{2}+z^{2}\right)^{3}}}+2 C_{2} C_{3} \frac{\kappa^{6} s^{2}}{\left(r_{\perp}^{2}+z^{2}\right)^{2}}\right]\right\} \\
& -\frac{12 i \kappa^{9} \sqrt{s} C_{1}^{3}}{(2 \pi)^{3}} \int d^{2} \mathbf{r}_{\perp} e^{i \mathbf{\Delta}_{\perp} \mathbf{r}_{\perp}}\left(\int_{-\infty}^{\infty} \frac{1}{\sqrt{r_{\perp}^{2}+z^{2}}} d z\right)^{3} .
\end{aligned}
$$

Perform some necessary calculations for the first term of Eq. (E.4), we have

$$
\begin{aligned}
J_{1} & =\frac{3 i \kappa^{6} \sqrt{s}}{(8 \pi)^{6}} F_{1}(t)+\frac{9\left(m_{1}+m_{2}\right)}{4(8 \pi)^{3} c^{2}} \frac{\kappa^{6} \sqrt{s}}{\sqrt{\mu^{2}-t}} \\
& +\frac{123 \kappa^{6} \hbar \sqrt{s}}{10(4 \pi)^{5} c^{3}} F_{2}(t),
\end{aligned}
$$

and the second term of Eq. (E.4) gives the result

$$
J_{2}=\frac{6 i \kappa^{10} \sqrt{s}}{(8 \pi)^{9}} F_{2}(t)
$$

The final result for the first correction term of the scattering amplitude has the form

$$
\begin{aligned}
& T_{\text {graviton }}^{(1)}(s, t)=J_{1}+J_{2} \\
& =\frac{3 i \kappa^{6} \sqrt{s}}{(8 \pi)^{6}} F_{1}(t)+\frac{9\left(m_{1}+m_{2}\right)}{4(8 \pi)^{3} c^{2}} \frac{\kappa^{6} \sqrt{s}}{\sqrt{\mu^{2}-t}} \\
& \quad+\frac{123 \kappa^{6} \hbar \sqrt{s}}{10(4 \pi)^{5} c^{3}} F_{2}(t)+\frac{6 i \kappa^{10} \sqrt{s}}{(8 \pi)^{9}} F_{2}(t) .
\end{aligned}
$$

\section{References}

1. G. 't Hooft, Phys. Lett. B 198, 61 (1987)

2. G. 't Hooft, Nucl. Phys. B 304, 867 (1988)

3. D. Amati, M. Ciafaloni, G. Veneziano, Phys. Lett. B 197, 81 (1987)

4. D. Amati, M. Ciafaloni, G. Veneziano, Phys. Lett. B 289, 87 (1992)

5. D. Amati, M. Ciafaloni, G. Veneziano, Int. J. Mod. Phys. A 3, 1615 (1988)
6. D. Amati, M. Ciafaloni, G. Veneziano, Nucl. Phys. B 347, 550 (1990)

7. D. Amati, M. Ciafaloni, G. Veneziano, Nucl. Phys. B 403, 707 (1993)

8. D. Amati, M. Ciafaloni, G. Veneziano, JHEP 0802, 049 (2008). arXiv:0712.1209 [hep-th]

9. C.O. Lousto, N. Sanchez, Phys. Lett. 232, 462 (1989)

10. M. Fabbrichesi, R. Pettorino, G. Veneziano, G.A. Vilkovisky, Nucl. Phys. B 419, 147 (1994)

11. R. Kirschner, Phys. Rev. D 52(4), 2333 (1995)

12. S. Kar, J. Manharana, Int. J. Mod. Phys. A 10, 2733 (1995)

13. M. Zeni, Class. Quantum Gravity 10, 905 (1993)

14. E. Verlinde, H. Verlinde, Nucl. Phys. B 371, 246 (1992)

15. D. Kabat, M. Ortiz, Nucl. Phys. B 388, 570 (1992)

16. S. Das, P. Majumdar, Phys. Rev. D 51, 5664 (1998)

17. S. Das, P. Majumdar, Phys. Lett. B 348, 349 (1995)

18. I. Muzinich, M. Soldate, Phys. Rev. D 37, 359 (1988)

19. Nguyen Suan Han, E. Ponna, Nuovo Cimento A 110, 459 (1997)

20. Nguyen Suan Han, Eur. Phys. J. C 16, 547 (2000)

21. Nguyen Suan Han, Nguyen Nhu Xuan, Eur. Phys. J. C 24, 643 (2002)

22. N.N. Bogoliubov, D.V. Shirkov, Introduction to the Theory of Quantized Fields (Wiley Interscience, New York, 1980)

23. E.S. Fradkin, Nucl. Phys. 76, 481 (1965)

24. E.S. Fradkin, Acta Phys. Hung. XIX, 176 (1965)

25. E.S. Fradkin, V. Esposito, S. Termini, Revista del Nuovo Cimento Serie I 2, 498-560 (1970)

26. G.A. Milekhin, E.S. Fradkin, JETP 45, 1926 (1963)

27. B.M. Barbashov, JEPT 48, 607 (1965)

28. H.M. Fried, Basics of Functional Methods and Eikonal Models, ed. by Frontieres (Gifsur-Yvette, 1990)

29. H. Abarbanel, C. Itzykson, Phys. Rev. Lett. 23, 53 (1969)

30. B.M. Barbashov, S.P. Kuleshov, V.A. Matveev, A.N. Sisakyan, A.N. Tavkhelidze, Phys. Lett. 33B, 419 (1970)

31. B.M. Barbashov, S.P. Kuleshov, V.A. Matveev, A.N. Sisakyan, A.N. Tavkhelidze, Phys. Lett. 33B, 484 (1970)

32. B.M. Barbashov, S.P. Kuleshov, V.A. Matveev, A.N. Sisakyan, Teor. Mat. Fiz, 3(3), 342 (1970)

33. B.M. Barbashov, S.P. Kuleshov, V.A. Matveev, A.N. Sisakyan, Teor. Mat. Fiz 5, 330 (1970)

34. V.N. Pervushin, Teor. Mat. Fiz. 4, 43 (1970)

35. V.A. Matveev, A.N. Tavkhelidze, Teor. Mat. Fiz 9, 44 (1971)

36. R. Blankenbecler, H.M. Fried, Phys. Rev. D 3, 678 (1973)

37. Nguyen Suan Han, V.N. Pervushin, Teor. Mat. Fiz 29, 178 (1976)

38. N.S. Han, L.A. Dung, N.N. Xuan, V.T. Thang. Int. J. Mod. Phys. A 31, 1650126 (2016)

39. A.A. Logunov, A.N. Tavkhelidze, Nuovo Cimento 29, 380 (1963)

40. A.A. Logunov, Nguyen Van Hieu, O.A. Khrustalev, Nucl Phys. 50, 295 (1964)

41. Nguyen Van Hieu, R.N. Faustov, Nucl. Phys. 53, 337 (1964)

42. V.A. Kadyshevsky, R.M. Mir-Kashimov, N.B. Skachkov, Nuovo Cimento 55A, 233 (1968)

43. V.R. Garvesevanishvili, V.A. Matveev, L.A. Slepchenko, A.N. Tavkhelidze, Phys. Lett. 29B, 191 (1969)

44. V.R. Garvesevanishvili, V.R. Goloskokov, V.A. Matveev, L.A. Slepchenko, Teor. Mat. Fiz. 1, 37 (1972)

45. I.T. Todorov, Phys. Rev. D 3, 2352 (1971). (Quasipotential Approach to the Two-Body Problems in Quantum Field Theory, IC/71/75)

46. M.A. Mestvirshvili, G.L. Rcheulishvili, Teor. Mat. Fiz. 8, 206 (1970)

47. S.P. Kuleshov, V.A. Matveev, M.V. Savelyev, A.N. Sissakian, M.A. Smondyev, Teor. Mat. Fiz. 6, 36 (1971)

48. A.B. Arbuzov, Nuovo Cimento 107A, 1263 (1994)

49. N.S. Han, L.T.H. Yen, N.N. Xuan. Int. J. Mod. Phys. A 27, 1250004 (2012) 
50. N.S. Han, N.N. Xuan, V.T. Thang, J. Phys. Sci. Appl. 7(4), 47 (2017)

51. I.F. Donoghue, Phys. Rev. Lett. 72, 2996 (1994). arXiv:gc-qc/9310024

52. I.F. Donoghue, Phys. Rev. D 50, 3874 (1994). arXiv:gc-qc/9405057

53. N.E.J. Bjerrum-Bohr, J.F. Donoghue, B.R. Holstein, Phys. Rev. D 67, 084033 (2003). [Erratum-ibid. D 71, 069903 (2005)]

54. J.F. Dononghue, T. Torma, Phys. Rev. D 54, 4963 (1996)

55. Y. Iwasaki, Prog. Theor. Phys. 46, 1587 (1971)

56. S. Deser, P. van Nieuwenhuizen, Phys. Rev. D 10, 401 (1974)

57. A. Shiekh, Can. J. Phys. 74, 172 (1996)

58. A. Akhundov, S. Bellucii, A. Shiekh, Phys. Lett. B 395, 16 (1997). arXiv:gr-qc/9611018

59. J.J. Muzinich, S. Volkos, Phys. Rev. D 52, 3472 (1995)

60. H. Hamber, S. Liu, Phys. Lett. 357 B, 51 (1995)

61. N.E.J. Bjerrum-Borh, Phys. Rev. D 66, 084023 (2002). arxiv:hep-th/0206236
62. I.B. Khriplovich, G.G. Kirilin, JETP 95, 981-986 (2002). arXiv:gr-qc/0207118

63. I.B. Khriplovich, G.G. Kirilin, JETP 98, 1063 (2004)

64. K.A. Kazakov, P.I. Pronin, Phys. Rev. D 62, 044043 (2000)

65. K.A. Kazakov, P.I. Pronin, Nucl. Phys. B 573, 536 (2000)

66. S. Faller, Phys. Rev. D 77, 124039 (2008)

67. E. Battista, Extreme Regimes in Quantum Gravity (Istituto Nazionale di Fisica Nucleare (INFN)-Sezione di Napoli, Napoli, 2016)

68. S.B. Giddings, R.A. Porto, The gravitational S-matrix. arXiv:0908.0004v1 [hep-th]

69. N.E.J. Bjerrum-Bohr, J.F. Donoghue, B.R. Holstein, L. Planté, P. Vanhove, Phys. Rev. Lett. 114, 061301 (2015)

70. A.S. Davydov, Quantum Mechanics (Pergamon Press, Oxford, 1965) 